\title{
Tunable Slow Light with Large Bandwidth and Low-dispersion in Photonic Crystal Waveguide Infiltrated with Magnetic Fluids
}

\author{
Weizheng Lei and Shengli Pu* \\ College of Science, University of Shanghai for Science and Technology, Shanghai 200093, China
}

(Received 6 February 2015, Received in final form 22 May 2015, Accepted 11 June 2015)

\begin{abstract}
Two kinds of magnetic fluids with different volume fractions are symmetrically filled into the W0.9 photonic crystal waveguide structure. The 2D plane-wave expansion method is used to investigate the slow light properties numerically. The constant group index criterion is employed to evaluate the slow light performance. The wavelength bandwidth $\Delta \lambda$ centering at $\lambda_{0}=1550 \mathrm{~nm}$ varies from 32.4 to $44.2 \mathrm{~nm}$ when the magnetic field factor $\alpha_{\|}$changes from 0 to 1 . And the corresponding normalized delay bandwidth product can be tuned from 0.221 to 0.258. For comparison and optimization, two infiltration cases are investigated and the more advantageous infiltration scheme is found.
\end{abstract}

Keywords : photonic crystal waveguides, slow light, tunability, magnetic fluid

\section{Introduction}

Photonic crystal waveguide is an effective means to achieve slow light, which can realize slow light with large and flexible bandwidth and thus has attracted great attention [1]. There are two main methods to obtain the tunable slow light with photonic crystal waveguides. One is to tune the slow light by adjusting the structure of the photonic crystal waveguide, such as engineering the hole shape or size, changing the period or direction of the photonic crystal lattice [2-5], using the coupled cavity or center hole in defect structure $[6,7]$. The other is to control the refractive index of liquid infiltrated into the holes of the photonic crystal structure, for instance, infiltrating the slotted photonic crystal waveguide with microfluidic technique [8].

The second method is superior to the first one because tuning the slow light can be realized by changing the refractive index of the infiltrated liquid easily and timely. Magnetic fluid is a good candidate liquid, whose refractive index can be adjusted by externally magnetic field and the volume fraction of magnetic nanoparticle $[9,10]$. For tuning the slow light, magnetic fluid is filled in the holes or used as the background of the photonic crystal

(C)The Korean Magnetics Society. All rights reserved.

*Corresponding author: Tel: +86-21-6566-7144

Fax: +86-21-6566-7144, e-mail: shlpu@usst.edu.cn structure placed in the magnetic field. Through changing the magnetic field, i.e. changing the refractive index of magnetic fluid, the slow light parameter of the structure can be set at the desired values. This kind of magnetically controllable optical component has been proved feasible in two-dimensional model [11] and photonic crystal slab configuration [12]. Recently, Jahanbakhshian et al. have studied the slow light properties in photonic crystal waveguide with asymmetric microfluidic infiltration [13]. Two kinds of materials with different refractive indices are infiltrated into the photonic crystal structure asymmetrically, which implies an alternative way to tune the slow light. In this work, the 3D photonic crystal slab waveguide filled with two kinds of magnetic fluids (different in volume fractions) is proposed and the corresponding slow light properties are investigated. The structure and infiltration parameters are optimized to obtain tunable slow light with large bandwidth and low dispersion.

\section{Waveguide Design}

The structure we proposed is silicon-based photonic crystal slab with triangular lattice. The lattice constant is $a$ and the radius of hole is $r=0.3 a$. The slab is sandwiched between silica and air with thickness of $h=283 \mathrm{~nm}$ (same to Ref. 11). Then, removing a row of holes and shifting other holes inward to form W0.9 line-defect waveguide. 

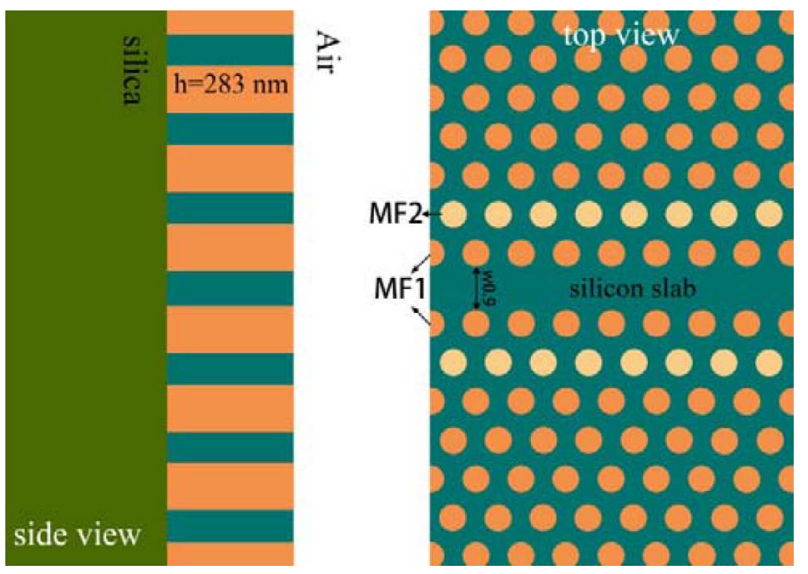

Fig. 1. (Color online) Schematic of the proposed photonic crystal waveguide structure. The lattice constant is $a$ and $r=$ 0.3a. Dark color of circle is $\mathrm{MF}_{1}$ and light color is $\mathrm{MF}_{2}$.

The details of waveguide structure are shown in Fig. 1. The magnetic fluid with volume fraction of $\phi_{2}$ and refractive index of $n_{2}$ (referred as $\mathrm{MF}_{2}$ ) is used to fill the second row of holes (see Fig. 1). The other rows of holes are filled with other magnetic fluid, whose volume fraction and refractive index are $\phi_{1}$ and $n_{1}$ (referred as $\mathrm{MF}_{1}$ ), respectively. Therefore, $\mathrm{MF}_{1}$ and $\mathrm{MF}_{2}$ are symmetrically filled into the photonic crystal structures on both sides of the line defect.

Through the Maxwell-Garnett approximation, the magnetic field dependent dielectric constant of magnetic fluid $\varepsilon_{M F}$ can be expressed as [14-17]

$$
\frac{\varepsilon_{M F}-\varepsilon_{\text {liq }}}{\alpha_{\|} \varepsilon_{M F}+\left(3-\alpha_{\|}\right) \varepsilon_{\text {liq }}}=\phi \frac{\varepsilon_{\text {particle }}-\varepsilon_{\text {liq }}}{\varepsilon_{\text {particle }}+2 \varepsilon_{\text {liq }}}
$$

where $\varepsilon_{l i q}$ is the dielectric constant of carrier liquid, $\varepsilon_{\text {particle }}$ is the dielectric constant of magnetic nanoparticle, parameter $\phi$ is the volume fraction of magnetic nanoparticles in magnetic fluid, parameter $\alpha_{\|}$means the degree of structural anisotropy caused by the magnetic field. $\alpha_{\|}$ changes in the range of 0-1 corresponding to the magnetic field varying from infinite to 0 . $\varepsilon_{\text {particle }}=2.2^{2}$ and $\varepsilon_{\text {liq }}=$ $1.448^{2}$ [12]. $\phi_{1}=0.5$ and $\phi_{2}=0.75$ are considered in current work. The magnetic permeability $\mu$ is taken as 1 at optical frequency. According to Eq. (1), the refractive indices of $\mathrm{MF}_{1}$ and $\mathrm{MF}_{2}$ are calculated out and listed in Table 1 and 2, respectively. The effective refractive index method based on 2D plane-wave expansion calculation is utilized to study the slow light performance $[18,19]$. The refractive index of silica, silicon and air are 1.45, 3.48 and 1 , respectively. Therefore, the equivalent slab effective index is obtained to be 3.0095 .
Table 1. Refractive index of $\mathrm{MF}_{1}$ for magnetic field factor $\alpha_{\|}$ varying from 0 to 1 . $\varepsilon_{\text {liq }}=1.448^{2}, \varepsilon_{\text {particle }}=2.2^{2}$ and $\phi_{1}=0.5$.

\begin{tabular}{ccccccc}
\hline \hline Magnetic field factor $\alpha_{\|}$ & 0 & 0.2 & 0.4 & 0.6 & 0.8 & 1 \\
\hline Refractive index $\mathrm{n}_{1}$ & 1.7469 & 1.7555 & 1.7645 & 1.7741 & 1.7843 & 1.7952
\end{tabular}

Table 2. Refractive index of $\mathrm{MF}_{2}$ for magnetic field factor $\alpha_{\|}$ varying from 0 to 1 . $\varepsilon_{\text {liq }}=1.448^{2}, \varepsilon_{\text {particle }}=2.2^{2}$ and $\phi_{2}=0.75$.

\begin{tabular}{ccccccc}
\hline \hline Magnetic field factor $\alpha_{\|}$ & 0 & 0.2 & 0.4 & 0.6 & 0.8 & 1 \\
\hline Refractive index $\mathrm{n}_{2}$ & 1.8787 & 1.8968 & 1.9165 & 1.9381 & 1.9618 & 1.9879
\end{tabular}

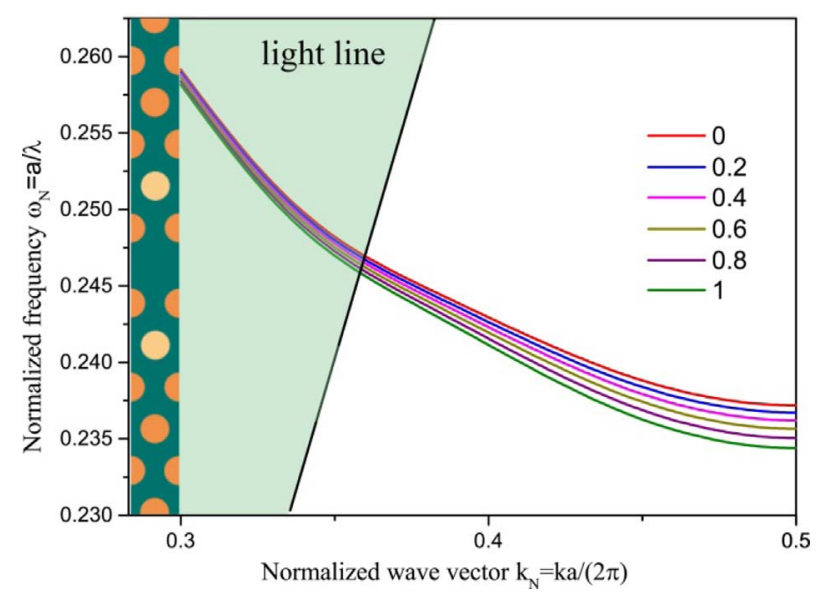

Fig. 2. (Color online) Dispersion curve of photonic crystal waveguide infiltrated with $\mathrm{MF}_{1}$ and $\mathrm{MF}_{2}$ symmetrically for magnetic field factor $\alpha_{\|}$varying from 0 to 1 . The supercell used for calculation is shown in the inset.

\section{Results and Discussion}

Using the data in Tables 1 and 2, the dispersion property of the guided mode in the photonic crystal waveguide is calculated and the result is shown in Fig. 2. Herein, the wave vector has been normalized as $k_{N}=k a$ l $2 \pi$, and the frequency has been normalized as $\omega_{N}=a / \lambda$. The light line of silica is also shown. Light can only propagate within the waveguide for the frequency under the light line. Fig. 2 indicates that the dispersion curve shifts to low frequency with the magnetic field factor $\alpha_{\|}$ increase from 0 .

According to Fig. 2, the group index $n_{g}=c \frac{d k}{d \omega}=\frac{d k_{N}}{d \omega_{N}}$ is calculated out. The corresponding results are illustrated in Fig. 3. Fig. 3 shows that $n_{g}$ is almost constant in the frequency range of $0.240<\omega_{N}<0.245$, which implies low-dispersion slow light.

According to $\beta_{2}=\frac{d^{2} k}{d \omega^{2}}=\frac{1}{c} \frac{d n_{g}}{d \omega}$, the group velocity dispersion (GVD) $\beta_{2}$ is obtained from Fig. 3. The corresponding $\beta_{2} \sim \omega_{N}$ curve is depicted in Fig. 4. Fig. 4 shows 


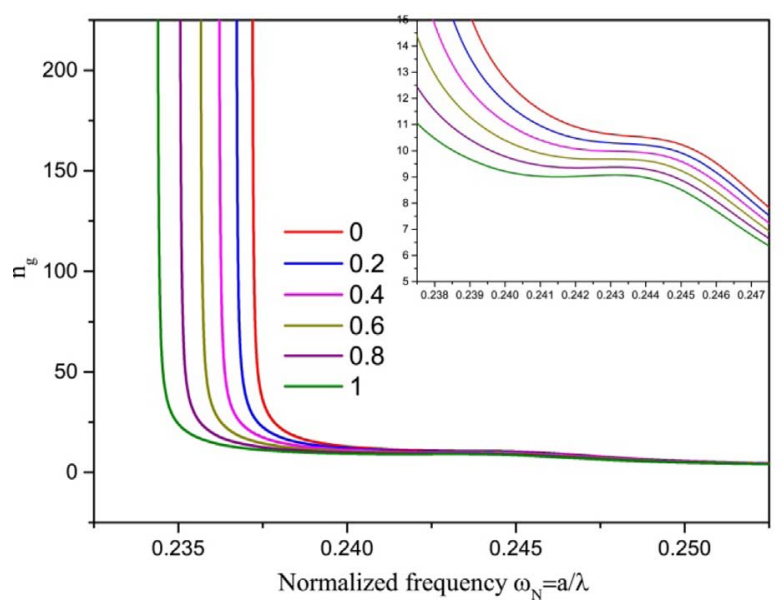

Fig. 3. (Color online) Group index of guided mode of the proposed structure at different magnetic fields.

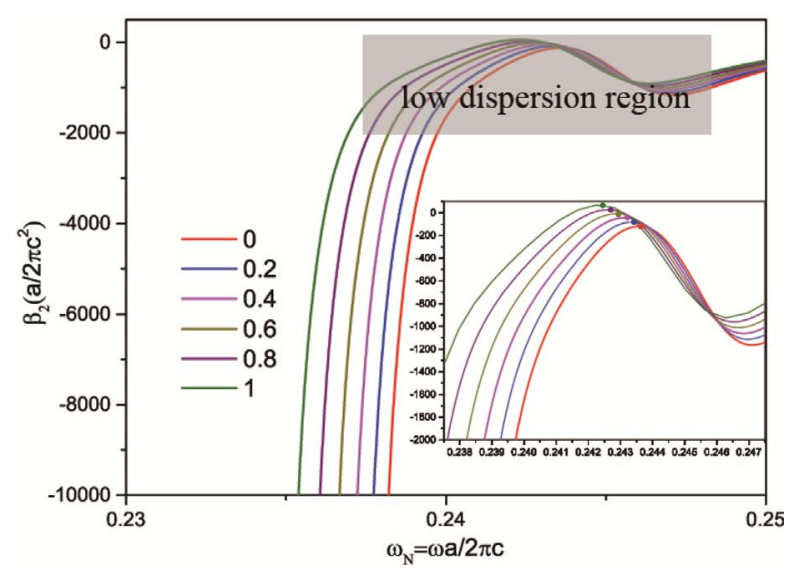

Fig. 4. (Color online) Group velocity dispersion of guided mode in photonic crystal waveguide filled with magnetic fluid for magnetic field factor $\alpha_{\|}$varying from 0 to 1 .

that there is a wide low-dispersion region with $-2 \times 10^{3} \mathrm{al}$ $\left(2 \pi c^{2}\right)<\beta_{2}<100 a /\left(2 \pi c^{2}\right)$. Comparing with the conventional low GVD definition, i.e. $\beta_{2}<10^{6} a\left(2 \pi c^{2}\right)$ [20], the absolute value of $\beta_{2}$ in our low-dispersion region is nearly three orders of magnitude smaller. Therefore, our lowdispersion criterion is much stricter than that usually employed.

In order to further describe the slow light properties quantitatively and comparably, the normalized delay bandwidth product NDBP $=n_{g} \cdot \frac{\Delta \omega}{\omega_{0}}=n_{g} \cdot \frac{\Delta \omega_{N}}{\omega_{N_{0}}}$ is employed, where $\omega_{N_{0}}$ is center frequency, $\Delta \omega_{N}=\omega_{H N}-\omega_{L N}$. Herein, the constant group index criterion is used [21]. Therefore, the center frequency $\omega_{N_{0}}$ is selected at the peak of GVD curve (see the inset of Fig. 4), which ensures the slow light in the whole bandwidth $\Delta \omega_{N}$ has the lowest dispersion. Then, the constant group index $n_{g}$

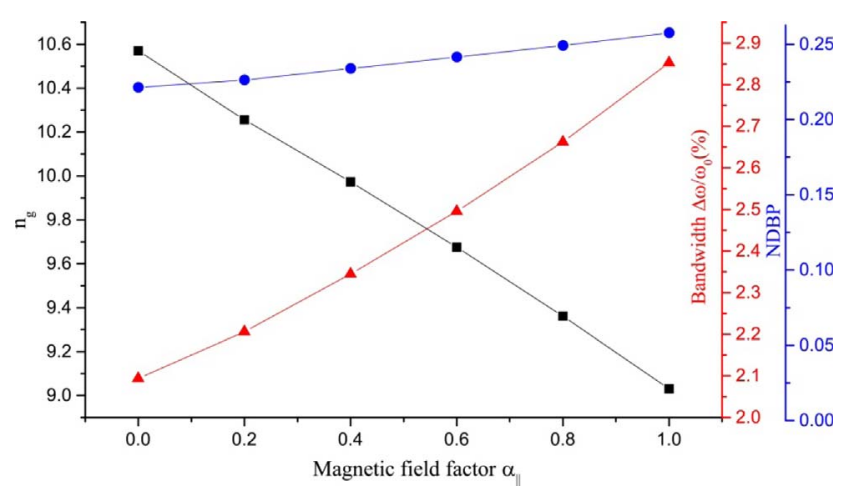

Fig. 5. (Color online) The relationship between magnetic field factor $\alpha_{\|}$and group index, NDBP, and normalized bandwidth.

corresponding to $\omega_{N_{0}}$ can be obtained from Fig. 3. $n_{g H}=$ $1.1 n_{g}$ and $n_{g L}=0.9 n_{g}$ corresponding to $\pm 10 \% n_{g}$ are calculated. Thus, the frequencies $\omega_{H N}$ and $\omega_{L N}$ corresponding to $n_{g H}$ and $n_{g L}$ are obtained from Fig. 3. The relationship between magnetic field factor $\alpha_{\|}$and group index $n_{g}$,

NDBP and normalized bandwidth $\frac{\Delta \omega_{N}}{\omega_{N_{0}}}$ is plotted in Fig.

5. Fig. 5 indicates that the NDBP and bandwidth can be tuned with the magnetic field factor in the range of 0.2210.258 and $2.09 \%-2.85 \%$, respectively. So, if the center wavelength is selected at $\lambda_{0}=1550 \mathrm{~nm}$, the wavelength bandwidth $\Delta \lambda$ can be tuned from 32.4 to $44.2 \mathrm{~nm}$ when magnetic field factor $\alpha_{\|}$changes from 0 to 1. Comparing with other optimized photonic crystal waveguides, our proposed photonic crystal waveguide has a much larger bandwidth $[5,22,23]$.

In order to be comprehensive, the opposite case (viz. the second row of holes is filled with $\mathrm{MF}_{1}$ and the other rows of holes are filled with $\mathrm{MF}_{2}$ ) is also investigated. Numerical results indicate that the opposite case has lower slow light performance than that of the aforementioned one.

To further investigate the infiltration-dependent slow light properties, the magnetic-field-dependent and magneticfield-independent $\Delta n=n_{2}-n_{1}$ are considered, respectively. For the magnetic-field-dependent $\Delta n$ case, the refractive indices listed in Table 1 and 2 are employed. According to Table 1 and 2, the relationship between $\Delta n$ and magnetic field is listed in Table 3. For the magneticfield-independent $\Delta n$ case, $\Delta n=n_{2}-n_{1}$ is set at 0.15 . The refractive indices listed in Table 1 is employed as $n_{1}$. So, $n_{2}=n_{1}+0.15$ as listed in Table 4 .

Table 3. $\Delta n$ at different magnetic field factors.

\begin{tabular}{ccccccc}
\hline \hline Magnetic field factor $\alpha_{\|}$ & 0 & 0.2 & 0.4 & 0.6 & 0.8 & 1 \\
\hline$\Delta \mathrm{n}=\mathrm{n}_{2}-\mathrm{n}_{1}$ & 0.1318 & 0.1413 & 0.1520 & 0.1640 & 0.1775 & 0.1927 \\
\hline
\end{tabular}


Table 4. $n_{1}$ and $n_{2}$ at different magnetic field factors when $\Delta n=0.15$.

\begin{tabular}{ccccccc}
\hline \hline Magnetic field factor $\alpha_{\|}$ & 0 & 0.2 & 0.4 & 0.6 & 0.8 & 1 \\
\hline Refractive index $\mathrm{n}_{1}$ & 1.7469 & 1.7555 & 1.7645 & 1.7741 & 1.7843 & 1.7952 \\
Refractive index $\mathrm{n}_{2}$ & 1.8969 & 1.9055 & 1.9145 & 1.9241 & 1.9343 & 1.9452 \\
\hline
\end{tabular}

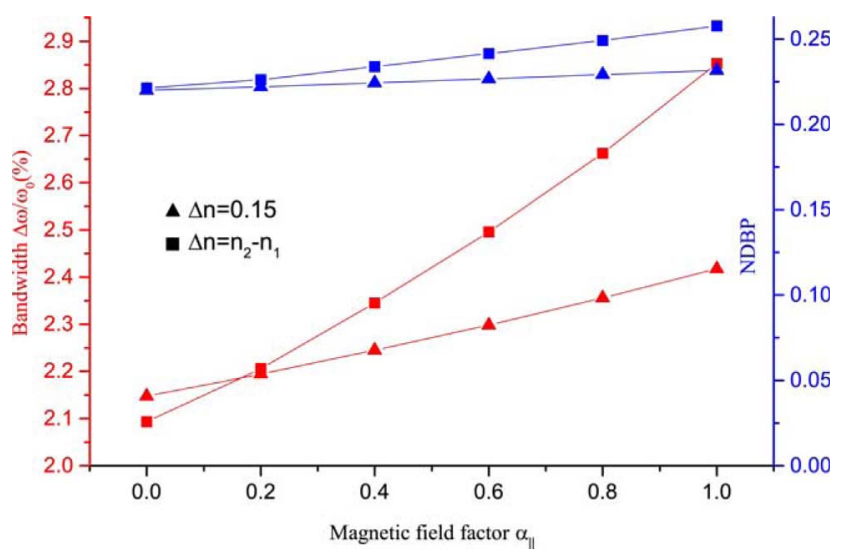

Fig. 6. (Color online) Bandwidth and NDBP for the magneticfield-dependent and magnetic-field-independent $\Delta n$. The magnetic field factor $\alpha_{\|}$varies from 0 to 1 .

The slow light performances for the two cases are calculated and the corresponding results are shown in Fig. 6. Fig. 6 indicates, in terms of bandwidth and NDBP, the tunable range for the magnetic-field-dependent $\Delta n$ case is larger than the magnetic-field-independent one. This implies that, besides changing refractive index of $\mathrm{MF}_{1}$ and $\mathrm{MF}_{2}$, changing $\Delta n$ with magnetic field is more favorable for tuning the slow light of the proposed structure in a wide bandwidth and simultaneously with a large NDBP.

\section{Conclusion}

In summary, the W0.9 photonic crystal waveguide is designed and two types of magnetic fluids are proposed to infiltrate the photonic crystal structure in order to realize tunable slow light with large bandwidth and low dispersion. The second row of holes is filled with magnetic fluid $\mathrm{MF}_{1}$ and other holes are filled with $\mathrm{MF}_{2}$. The effective refractive index method based on $2 \mathrm{D}$ plane-wave expansion calculation is employed. The constant group index criterion is used to study the slow light properties. With the magnetic field factor $\alpha_{\|}$varying from 0 to 1 , the bandwidth can be tuned from 32.4 to $44.2 \mathrm{~nm}$ and NDBP can be tuned from 0.221 to 0.258 . Besides, comparing with the magnetic-field-independent $\Delta n$ case, tunable range of bandwidth and NDBP for the magnetic-fielddependent $\Delta n$ case is superior. The obtained results imply the advantages of the proposed structure, viz. tunable, low dispersion and high NDBP slow light.

\section{Acknowledgments}

This research is supported by Natural Science Foundation of Shanghai (No. 13ZR1427400), Shanghai Key Laboratory of Specialty Fiber Optics and Optical Access Networks (Grant No. SKLSFO2014-05), and Hujiang Foundation of China (B14004).

\section{References}

[1] J. B. Khurgin and R. S. Tucker. Slow Light: Science and Applications, CRC, Boca Raton (2009). pp. 59-75.

[2] Y. Wan, Y. Guo, Y. Zhang, and M. Yun, Opt. Appl. 43, 831 (2013).

[3] K. Zhu, T. Deng, Y. Sun, Q. Zhang, and J. Wu, Opt. Commun. 290, 87 (2013).

[4] J. Tang, T. Wang, X. Li, B. Liu, B. Wang, and Y. He, J. Opt. Soc. Am. B 31, 1011 (2014).

[5] B. Meng, L. Wang, X. Li, W. Xiao, L. Wang, and D. Xiang, Opt. Commun. 285, 3704 (2012).

[6] K. Zhu, T. Deng, Y. Sun, Q. Zhang, and J. Wu, Opt. Commun. 285, 2611 (2012).

[7] K. Üstün and H. Kurt, ICTION Conf. Proc. 14, 1 (2012).

[8] J. Wu, Y. Li, C. Peng, and Z. Wang, Opt. Commun. 284, 2149 (2011).

[9] H. E. Horng, C.-Y. Hong, S. Y. Yang, and H. C. Yang, Appl. Phys. Lett. 82, 2434 (2003).

[10] H. Wang, S. Pu, N. Wang, S. Dong, and J. Huang, Opt. Lett. 38, 3765 (2013).

[11] S. Pu, H. Wang, N. Wang, and X. Zeng, Appl. Phys. B, 112, 223 (2013).

[12] S. Pu, S. Dong, and J. Huang, J. Opt. 16, 045102 (2014).

[13] M. Jahanbakhshian and R. Karimzadeh, Laser Phys. 24, 105903 (2014).

[14] C. Fan, G. Wang, and J. Huang, J. Appl. Phys. 103, 094107 (2008).

[15] J. Huang, and K. Yu, Phys. Rep. 431, 87 (2006).

[16] C. Fan and J. Huang, Appl. Phys. Lett. 89, 141906 (2006).

[17] Y. Gao, J. P. Huang, Y. M. Liu, L. Gao, K. W. Yu, and X. Zhang, Phys. Rev. Lett. 104, 034501 (2010).

[18] M. Qiu, Appl. Phys. Lett. 81, 1163 (2002).

[19] D. Gao and Z. Zhou, Appl. Phys. Lett. 88, 163105 (2006).

[20] J. Tang, T. Wang, X. Li, B. Wang, C. Dong, L. Gao, B. Liu, Y. He, and W. Yan, J. Lightwave Technol. 31, 3188 (2013).

[21] Y. Xu, L. Xiang, E. Cassan, D. Gao, and X. Zhang, Appl. Opt. 52, 1155 (2013).

[22] V. Varmazyari, H. Habibiyan, and H. Ghafoorifard, SOP Trans. Appl. Phys. 1, 55 (2014).

[23] M. Janfaza and M.-A. Mansouri-Birjand, Opt. Commun. 333, 58 (2014). 